\title{
In-Body Backscatter Communication and Localization
}

\author{
Deepak Vasisht ${ }^{1}$, Guo Zhang ${ }^{1}$, Omid Abari ${ }^{2}$, Hsiao-Ming Lu ${ }^{3}$, Jacob Flanz ${ }^{3}$, Dina Katabi ${ }^{1}$ \\ ${ }^{1}$ MIT CSAIL, ${ }^{2}$ University of Waterloo, ${ }^{3}$ Massachusetts General Hospital
}

\begin{abstract}
Backscatter requires zero transmission power, making it a compelling technology for in-body communication and localization. It can significantly reduce the battery requirements (and hence the size) of micro-implants and smart capsules, and enable them to be located on-the-move inside the body. The problem however is that the electrical properties of human tissues are very different from air and vacuum. This creates new challenges for both communication and localization. For example, signals no longer travel along straight lines, which destroys the geometric principles underlying many localization algorithms. Furthermore, the human skin backscatters the signal creating strong interference to the weak in-body backscatter transmission. These challenges make deep-tissue backscatter intrinsically different from backscatter in air or vacuum. This paper introduces ReMix, a new backscatter design that is particularly customized for deep tissue devices. It overcomes interference from the body surface, and localizes the in-body backscatter devices even though the signal travels along crooked paths. We have implemented our design and evaluated it in animal tissues and human phantoms. Our results demonstrate that ReMix delivers efficient communication at an average SNR of $15.2 \mathrm{~dB}$ at $1 \mathrm{MHz}$ bandwidth, and has an average localization accuracy of $1.4 \mathrm{~cm}$ in animal tissues.
\end{abstract}

\section{ACM Reference Format:}

Deepak Vasisht, Guo Zhang, Omid Abari, Hsiao-Ming Lu, Jacob Flanz, Dina Katabi. 2018. In-Body Backscatter Communication and Localization. In SIGCOMM '18: ACM SIGCOMM 2018 Conference, August 20-25, 2018, Budapest, Hungary. ACM, New York, NY, USA, 15 pages. https://doi.org/10.1145/3230543.3230565

\section{INTRODUCTION}

Backscatter is one of the most power-efficient communication technologies [24, 37]. It allows a device to communicate at zero power budget, simply by reflecting an ongoing RF signal. The reflected signal may also be used to localize the backscatter device. Because of these properties, there is much interest

Permission to make digital or hard copies of all or part of this work for personal or classroom use is granted without fee provided that copies are not made or distributed for profit or commercial advantage and that copies bear this notice and the full citation on the first page. Copyrights for components of this work owned by others than ACM must be honored. Abstracting with credit is permitted. To copy otherwise, or republish, to post on servers or to redistribute to lists, requires prior specific permission and/or a fee. Request permissions from permissions@acm.org.

SIGCOMM '18, August 20-25, 2018, Budapest, Hungary

(C) 2018 Association for Computing Machinery.

ACM ISBN 978-1-4503-5567-4/18/08 . . \$15.00

https://doi.org/10.1145/3230543.3230565

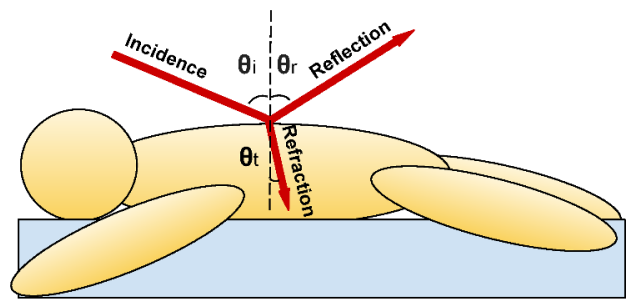

Figure 1-A large portion of RF signal is reflected off the human body. The signal that crosses the interface changes direction.

in using backscatter to localize and communicate with microimplants in the human body $[22,42,66,67]$. In particular, we have recently witnessed the introduction of implantable RFID microchips, the size of a grain of rice, which can be injected in the body using a needle. Such RFIDs have been deployed in animals and humans for identification, tracking, sensing, and even replacing one's own credit card [7, 41, 42].

Today, RFID implants are inserted directly under the skin, within a few mm from the body surface. But, if backscatter could be made to work much deeper in the body (e.g., $3-10 \mathrm{~cm}$ ), it will enable multiple critical applications. In particular, swallowable capsule endoscopes (e.g., PillCam) have made examining the gastrointestinal (GI) tract much easier by eliminating the pain associated with inserting conventional scopes. Backscatter can address two key challenges with existing capsules. Today half the capsule's energy is spent on RF transmission [65]. Backscatter can significantly reduce the power budget of these capsules, allowing them to function for a longer time [2]. Further, if the backscatter signal can be localized accurately (to within a couple of $\mathrm{cm}$ ), it would enable the capsule to adapt its functions depending on its location in the GI tract. For example, it can deposit drugs in certain areas, or adapt video frame rate to obtain higher resolution at critical areas $[49,58]$. Other potential applications of deep-tissue backscatter involve tracking micro-robots in blood stream [66,67], and localizing fiducial markers to detect movements of breast, liver or lung tumors during radiation therapy $[25,34]$.

However, deep-tissue backscatter is challenging because:

- Surface Interference: Due to the large difference in electrical properties between the human body and air, a large portion of the incident RF signal is reflected off the human surface, as shown in Fig. 1. Furthermore, RF signals experience exponentially more attenuation in human tissues (e.g., muscles) than in air. Thus, if the implant is few centimeters below the surface, the RF signal loses about $40 \mathrm{~dB}$ each way compared to reflections off the skin. As a result, the backscatter signal is $80 \mathrm{~dB}$ ( 100 million times) weaker than the signal reflected off the body surface. This causes a dynamic range problem at the receiver - i.e., the receiver ADC will be saturated by 
the larger reflection from the skin level and unable to detect the backscatter signal.

- Signal Deflection: RF signals propagate 8 times slower in muscles than in air. This difference breaks past algorithms for RF-based localization. A key principle in localization is that signals propagate along straight lines, and hence (in the absence of multipath), the direction of the signal is the same as the direction of the source. Yet, the difference in speed between air and muscle causes the signal to significantly deflect ( Fig. 1) as it enters the body.

This paper introduces ReMix, a novel wireless system for deep-tissue backscatter. A key property of ReMix is its ability to address the above challenges -i.e., it can capture the backscatter signal and localize a deep-tissue device despite surface interference and signal deflection.

So, how does one isolate the signal from a backscatter implant, when skin reflections are 8 orders of magnitude stronger than the backscattered signal. Our insight is to use non-ideal behavior in circuits to separate the backscatter reflections from all other reflections in the environment. Specifically, we rely on the concept of non-linearity. Standard circuits are designed to have linear behavior in their operating range, i.e., if the input receives signal at two different frequencies $f_{1}$ and $f_{2}$, the output transmits frequencies $f_{1}$ and $f_{2}$. In contrast, non-linear behavior (which is non-ideal) mixes the input frequencies, i.e, given input signal at frequencies $f_{1}$ and $f_{2}$, the output signal contains the original frequencies $f_{1}$ and $f_{2}$, along with linear combinations such as $f_{1}+f_{2}, f_{1}-f_{2}, 2 f_{1}-f_{2}$, etc. In traditional RF design, these combinations are unwanted and designers suppress them as much as possible. In contrast to the traditional approach, our insight is that we can use this unwanted behavior to our advantage and isolate the backscatter reflections.

Specifically, instead of using a standard backscatter circuit on the implant, we use a circuit that promotes non-linearities. In this case, the backscatter circuit mixes the input frequencies and reflects both the original frequencies as well as the various mixes. We can then tune our receiver to listen to the mixed frequencies, e.g., $f_{1}+f_{2}, 2 f_{1}-f_{2}$, etc. Since skin reflections are at $f_{1}$ and $f_{2}$, they can be filtered out. Thus, the backscatter signals at mixed frequencies like $f_{1}+f_{2}$ and $2 f_{1}-f_{2}$ are interference free. In $\$ 5$, we explain how we implement such non-linearity while keeping the RFID circuit passive (i.e., no power source).

Second, we need to localize the backscatter implant even though the signal travels along crooked paths. To address this problem, we introduce a new model for RF-based localization where the path between two points is modeled with linear splines (piecewise segments) as opposed to straight lines. The length of each segment refers to the stretch of the path in a particular material (air, fat, muscle). We analyze the model given the electrical properties of human tissues, which reveals interesting properties of in-body propagation such as: (a) one can ignore in-body multi-path without affecting accuracy; and (b) while the implant antenna radiates signal in the body in all directions, due to the difference in muscle electrical properties, all signals that leave the body exit through a small area on the surface. By leveraging such in-body propagation properties, we design an accurate localization system that accounts for the fact that the signal suffers deflection and has a different propagation speed (i.e. different wavelength) in different body tissues. Our model also accounts for the signal changing frequency inside the body - i.e. it enters the body as $f_{1}$ and $f_{2}$, gets mixed at the implant, and continues as $f_{1}+f_{2}, 2 f_{1}-f_{2}$, etc.

We have implemented ReMix and evaluated its performance using both animal tissues (chicken and pork) and human tissuephantoms, which are designed to emulate human tissue electrical properties. This is in-line with evaluations used for in-body applications like ultrasound imaging [13], in-body wireless power transfer [2], tomographic imaging [44, 51], etc. Our results can be summarized as follows:

- ReMix delivers effective backscatter communication. It achieves an SNR between $11.5 \mathrm{~dB}$ and $17 \mathrm{~dB}$ (for $1 \mathrm{MHz}$ bandwidth) for a backscatter device that is 1 to $8 \mathrm{~cm}$ deep in animal tissues. This performance is due to ReMix's non-linear mixing of the RF signals which allows it to deliver good SNRs despite strong surface interference.

- ReMix delivers accurate backscatter localization. Its algorithm can localize a deep-tissue implant to an average accuracy of $1.4 \mathrm{~cm}$. In contrast, directly applying standard localization algorithms results in an average error of $7.5 \mathrm{~cm}$ due to inability to deal with signal deflection.

Contribution: This paper has the following contributions:

- It is the first to demonstrate deep-tissue backscatter communication and localization in phantom and animal tissues.

- It is the first paper that proposes the use of non-linearities to deal with interference from unwanted skin reflections that can mask the desired signal from the in-body implant.

- It is the first to present a time-of-flight localization algorithm that accounts for signal deflection in body tissues.

\section{BACKGROUND AND RELATED WORK}

The medical industry is looking at a wide array of in-body devices that include pacemakers that communicate their data over the wireless channel, smart pills that image the gastrointestinal tract, and microscale robots that access organs through the bloodstream. Today, such deep tissue systems communicate by generating their own radio signal, a process that consumes a lot of energy. For instance, in wireless capsule endoscopes, RF consumes 4 to 10 times more power than the sensors [65]. As a result, these capsules use large batteries that occupy about $40-50 \%$ of the space of the capsule [5, 9]. Reducing the power requirement for $\mathrm{RF}$ transmissions can reduce the size of the capsules making them more easy to swallow. It can also improve completion likelihood. Past work has found that $16.5 \%$ 
of the times, capsule endoscopes fail to completely visualize the small bowel primarily due to limited battery life [48].

Similarly, interest in deep tissue localization is on the rise. Localization of deep tissue sensors like capsule endoscope can enable physicians to isolate the parts of the GI tract with abnormalities, adapt video frame rates based on location, and deposit biomarkers at specific locations $[49,58]$. The localization requirements for such capsules are on the order of a few centimeters [49].

Past work has tried to tackle these problems along multiple axes. Researchers have considered wireless power transfer -i.e., charging an implant using RF signals [2, 39]. These systems typically operate in the midfield where the RF transmitter is either directly in touch with the body or within a few centimeters from it. Our work is complementary to this literature. First, we address localization which is not addressed by power transfer. Second, even if the implant has the ability to harvest power, it can still leverage backscatter to communicate at zero power and save its harvested energy for its sensing tasks.

The literature also has few proposals for in-body localization. One line of research uses magnetic field analysis [4, 23, 49]. The advantage of using the magnetic field is that its properties do not change much between air and human tissues. The disadvantage however is that the magnetic field power decays with a factor $d^{6}$ as it travels through air [12]. Hence, the magnetic receiver (the receiving coil) has to be in touch with the body surface or within a few centimeters. Further, magnetic implants can be problematic. They can be painful if the person is exposed to a strong magnetic field as in the case of MRI [29]. They can also affect affect MRI images making it difficult to detect a tumor in the area near the implant [29]. A a result, this form of localization is not widely used. Doctors also use $\mathrm{X}$-ray or sonar for localization. These methods are expensive. Further, continuous tracking of an implant requires excessive $\mathrm{x}$ ray exposure which increases cancer risk [14]. Finally, the use of ultrasound for in-body localization requires direct contact with the human skin, making it infeasible for several medical applications. For instance, presence of metallic equipment close to the human body can be an hindrance for administering X-ray/proton beams used for radiation therapy in cancer treatments $[15,45]$.

Many implants need RF signals to communicate their data. It would be highly beneficial if the same signal can also be used for localization. Past proposals for in-body RF-based localization use the received signal strength (RSS) [62, 64]. Those systems use an array of receive antennas and either assume the implant to be closest to the receive antenna with the highest power or use path loss models to estimate location [58]. Analysis of the error bounds on RSS in-body localization has reported lower bounds of 4 to $6 \mathrm{~cm}$ [64] even when using up to 50 receive antennas. Past work has also tried to adapt indoor localization based on time-of-flight (ToF) or angle of arrival (AoA) for the domain [8, 40, 59]. Unfortunately, these systems are based purely on simulation, lack any empirical results, and most of them ignore signal deflection.

There is also a rich literature about backscatter communication and localization in-air [6, 24, 37, 68]. We build on this foundation but focus on intrinsically different problems that stem from RF propagation in deep tissues, such as signal deflection and body surface interference. We note that the design in $[24,30,68]$ proposes shifting the frequency of the backscatter signal to avoid WiFi interference. While ReMix also shifts the backscatter frequency, it differs in both goal and technique. ReMix's aim is to avoid interference from the skin surface, and its solution is completely passive and does not require any active components on the implant.

Finally, past work on harmonic RADARs and RFID-based localization ( $[10,20,21,50])$ has used non-linearity to mix two frequencies and weed out unwanted reflections from the environment. However, ReMix is the first system to use this technique to separate skin reflections from the signal from inbody implants. Furthermore, as described before, ReMix solves unique localization challenges (like signal deflections, change of wavelength in-body) that do not exist in in-air localization.

\section{RF SIGNALS IN BODY AND IMPLICATIONS FOR BACKSCATTER}

We start with an analysis of how RF signals behave as they propagate in biomaterial (e.g., fat, muscles), and the implications for in-body backscatter. From the perspective of electromagnetic (EM) waves, each material is characterized by two parameters: relative electrical permittivity, $\epsilon_{r}$ and relative magnetic permeability, $\mu_{r}$. These are complex numbers that capture how the electrical and magnetic fields in an EM wave interact with the material. Both $\epsilon_{r}$ and $\mu_{r}$ are 1 for air and vacuum. For biological tissues, the relative magnetic permeability $\mu_{r}$ can be approximated as 1 [32], so we set $\mu_{r}=1$ for the rest of the paper. However, $\epsilon_{r}$ has high variability depending on the tissue type and frequency of transmission. For example, for frequencies around $1 \mathrm{GHz}$ (commonly used by in-body implants), the value of $\epsilon_{r}$ in muscle is $55-18 j$ [26].

The value of $\epsilon_{r}$ is very important because it changes the speed of light and other electromagnetic waves (EM) in a material. Specifically, the speed of light in a biomaterial (e.g., muscle, fat, skin) is given by: $v=\frac{c}{\sqrt{\epsilon_{r}}}$, where $c$ is the speed of light in vacuum and (to a good approximation) air. The change in the speed of the EM wave has important implications.

(a) Attenuation Increases Exponentially: Recall that, for a signal at frequency $f$, traveling in free space from a transmitter to a receiver separated by distance $d$, the wireless channel $h(f, d)$ is given by

$$
h(f, d)=\frac{A}{d} e^{-j 2 \pi f \frac{d}{c}}
$$

where $A$ is the attenuation constant that depends on the antenna beam patterns and $c$ is the speed of light in vacuum. 


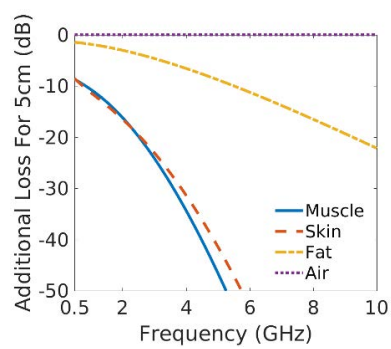

(a) Attenuation

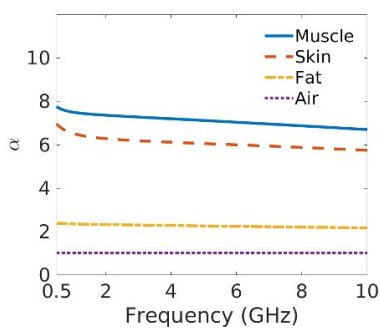

(b) Phase Change

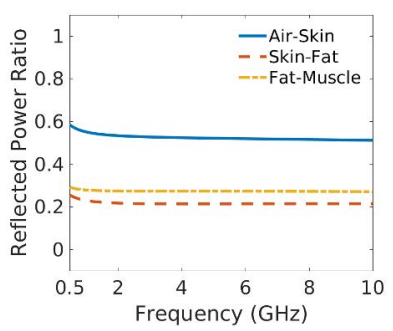

(c) Reflection

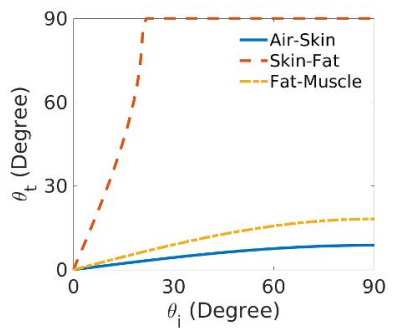

(d) Refraction

Figure 2-Signal Change in Human Body: As RF signals traverse human body, they suffer (a) additional attenuation, (b) increased phase change, and (c) reflection and (d) refraction at the interfaces.

For biomaterial, incorporating EM wave speed change in equation (1) gives us the wireless channel, $h_{M}(f, d)$ :

$$
h_{M}(f, d)=\frac{A}{d} e^{-j 2 \pi f \frac{d \sqrt{\epsilon}}{c}}
$$

To understand the impact of $\epsilon_{r}$ on wave propagation, let us write $\sqrt{\epsilon_{r}}=\alpha-\beta j$, where $\alpha$ and $\beta$ are positive real numbers. The channel equation can then be updated as:

$$
h_{M}(f, d)=\frac{A}{d} e^{-j 2 \pi f \frac{d(\alpha-\beta j)}{c}}=\frac{A}{d} e^{-j 2 \pi f \frac{d \alpha}{c}} e^{-2 \pi f \frac{d \beta}{c}}
$$

Note that the term $e^{-2 \pi f \frac{d \beta}{c}}$ causes exponential loss in magnitude of the signal during propagation. The higher the value of $\beta$, the higher the loss. This is in addition to the propagation attenuation experienced by the signal in free space, given by $\frac{A}{d}$. Fig. 2(a) plots the additional loss observed by an EM wave traveling for $5 \mathrm{~cm}$ in different biomaterials, i.e. muscle, fat and skin. As can be seen from the figure, muscle tissues and skin tissues are similar to each other but are very different from fat, which is closer to air. Also, muscle tissues experience significant additional loss in comparison with in-air signals.

There are two take home messages from Fig. 2(a). First, inbody RF signals should use relatively low frequencies to avoid the drastic power loss occurring at higher frequencies. In fact, it is a common practice to use frequencies about $1 \mathrm{GHz}$, which are small enough to have a relatively low loss, but also large enough to enable relatively small electronics and antennas [2, 39]. Second, for backscatter signals which have to traverse the body twice, they lose more than $20 \mathrm{~dB}$ just to get $5 \mathrm{~cm}$ deep. This amount is important as we start accumulating the various losses that eventually lead to about $80 \mathrm{~dB}$ of surface to backscatter interference (discussed in detail in §5.1).

(b) Antennas become less efficient: The electrical permittivity of a material further affects the efficiency of in-body antennas. As an antenna is placed in-body, its radiation efficiency decreases and its inherent losses increase as a function of $\epsilon_{r}$. For muscle tissues, these effects incur another $10-20 \mathrm{~dB}$ of loss depending on the antenna design [31].

(c) Wavelength Shrinks: Consider again Eq. 3. Note that the signal phase changes much faster in biomaterial than in air. Specifically, the phase changes $\alpha$ times faster in biomaterial than in air. This is because the wavelength is $\alpha$ times smaller.
Fig. 2(b) plots $\alpha$ for different materials inside the human tissues, i.e. muscle, fat and skin. It shows that the phase changes 8 times faster in muscle than air. This property is useful for RFbased localization algorithms that leverage phase changes to measure distance because it increases sensitivity and allows for measuring smaller distances (for the same signal SNR).

(d) Signal Reflection: The value of $\epsilon_{r}$ affects not only how the signal travels through a material, but also affects what happens at the interface between two materials. Consider a signal traveling from a material with relative permittivity $\epsilon_{r 1}$ to a material with relative permittivity $\epsilon_{r 2}$. Further, for ease of exposition, assume that the signal is traveling perpendicular to the interface, which is the direction of minimum reflection (the more general case is discussed in [47].) In this case, the ratio of the reflected power $P_{r}$ and incident power $P_{t}$ is given by:

$$
\frac{P_{r}}{P_{t}}=\left|\frac{\sqrt{\epsilon_{r 1}}-\sqrt{\epsilon_{r 2}}}{\sqrt{\epsilon_{r 1}}+\sqrt{\epsilon_{r 2}}}\right|^{2}
$$

As can be seen in the equation, larger the difference between the properties of two materials, the more signal power is reflected. Fig. 2(c) plots the ratio of power reflected for different interfaces as a function of frequency. Since going in-body requires the signal to traverse air-skin, skin-fat and fat-muscle interfaces (twice for backscatter), a large portion of the power is reflected back before it reaches the implant.

(e) Signal Refraction: When an RF signal traverses the interface between two materials, it experiences a change in direction, as shown in Fig. 1. This bending in the signal is called refraction. The relationship of the angle of incidence $\left(\theta_{i}\right)$ and the angle of refraction $\left(\theta_{t}\right)$ can be approximated by the following equation (for exact equation, see [47]):

$$
\operatorname{Re}\left(\sqrt{\epsilon_{r 1}}\right) \sin \theta_{i}=\operatorname{Re}\left(\sqrt{\epsilon_{r 2}}\right) \sin \theta_{t}
$$

where $\operatorname{Re}($.$) denotes the real part of a complex number. We$ plot the angle of refraction for different angles of incidence for various interfaces in Fig. 2(d). The figure shows that RF signals experience significant bending at skin-fat, fat-muscle and airskin interfaces. This is the key challenge for localization of a device implanted inside the body.

We make an important observation related to the air-skin interface, i.e., the blue line in Fig. 2(d). The figure clearly 


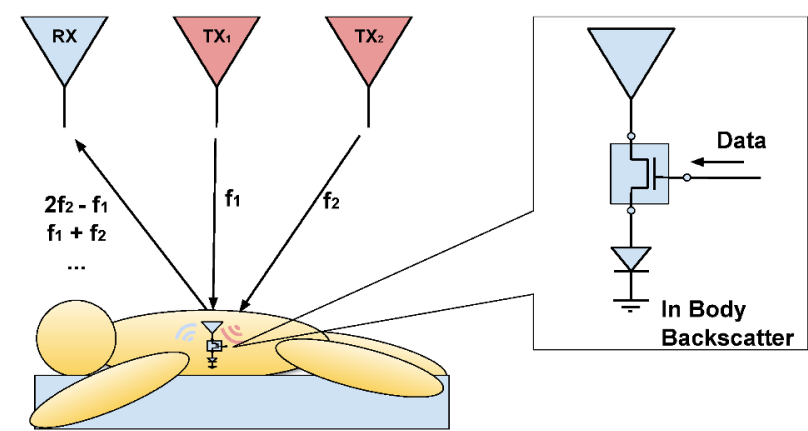

Figure 3 -An overview of ReMix's system setup and a schematic of the backscatter device (inlet).

shows that regardless of the incident angle, the refraction angle is always near zero. This means that it does not matter how the signal arrives in air, it enters the body almost along the direction of the normal on the surface (Fig. 1). Since EM wave paths are reversible, this result means that all $\mathrm{RF}$ waves that exit the body must arrive at the skin-air interface almost perpendicular to the body surface. RF waves that try to exit the body with an angle far from the normal are not allowed to do so, and are reflected back inside the body. We will revisit this observation later as we talk about RF-based in body localization.

Finally, we end by reminding the reader that properties (a), (b) and (d) above all combine to reduce the power of the backscatter signal and contribute to the surface interference problem. Properties (c) and (e) on the other change the signal's path and wavelength and hence affect localization.

\section{ReMix System SetuP}

ReMix is a backscatter design for deep-tissue implants or sensors (e.g., smart capsule). ReMix has two main components: a transceiver system comprising of multiple antennas and an in-body backscatter module (i.e., RFID). The transceiver system has two transmit antennas, one for each frequency being transmitted and multiple receive antennas.

This setup can be placed below or above a person's bed or on the side. The transceiver does not need to touch the body. It works in the far field and can be anywhere between 0.5 meters to a few meters. The in-body module is a small unit that can be attached to standard in-body devices that need to communicate data or be localized. An example arrangement is presented in Fig. 3. While it is common to perform medical procedures while the patient is lying on a bed, the operation of ReMix does not require the patient to be in a particular position.

\section{In-Body Backscatter Communication}

We discuss the challenges that face in-body backscatter communication and how ReMix overcomes them.

\subsection{Understanding Surface Interference}

As explained earlier, the signal reflected off the surface of the human body is much more than the small backscatter signal from a deep tissue device. To understand the power ratio between these two signals, let us consider a transceiver placed outside the human body and compare the reflected power observed from human body with the reflected power from an antenna in deep tissue ( $5 \mathrm{~cm}$ below the skin), connected to a perfect backscatter system (i.e., no loss in the backscatter circuit).

There are four major sources of power differences between skin reflections and reflections from the backscatter device. First, as discussed in $\$ 3$, a portion of the incident power is reflected at the interface between air and body, and thus never ends up reaching the backscatter system. Second, since the human body is a lossy medium, the signal faces large attenuation (exponential in distance) during in-body propagation (see Eq. 3). Third, the antenna efficiency in-body is reduced by $10-20 \mathrm{~dB}$ [31]. Finally, the effective area of radiation of an in-body antenna is much smaller than the skin area.

We do a back of the envelope calculation to combine these factors and compare the power reflected off the skin with the power reflected by the backscatter device. The attenuation, reflection and antenna efficiency loss from $\$ 3$ produce a combined loss of at least $30 \mathrm{~dB}$ in one direction. Since backscatter is two way, the total loss is at least $60 \mathrm{~dB}$. When we include the effect of the effective area of the skin which is much larger than an in-body antenna (typically the size of grain of rice [19]), the total loss is $\approx 80 \mathrm{~dB}$. Similar loss has been empirically observed by several experiments in past work $[2,35]$.

Thus, the signal reflection measured from the backscatter system is at least $80 \mathrm{~dB}$ lower than the signal measured from the surface. Such a huge difference in power will overwhelm the receiver's ADC and prevent it from capturing the backscatter signal. The problem is exacerbated by the fact that the human body is not static. Breathing, pulsing, and bowel movements cause the skin to move and vibrate. As a result the signal reflected by the body surface changes in unpredictable way. Thus traditional approaches used to eliminate self-interference in backscatter systems do not apply. ${ }^{1}$

\subsection{Exploiting Non-Linear Behavior}

How does one go about isolating the signal received from inside the body when the skin reflections are $80 \mathrm{~dB}$ (100 million times) stronger than the signal from inside the body? One approach to solve this problem is to enable the in-body backscatter device to shift the frequency. If the signal reflection from the skin and the in-body device are received at sufficiently different frequencies, one can filter the skin reflections out and receive just the backscatter signal.

In state-of-the-art backscatter systems ([24, 30, 68]), the standard way to shift frequencies is to use a small battery and

\footnotetext{
${ }^{1}$ Gating methods used in radar to reject nearby reflections do not apply because the distance between implant and skin is only a few $\mathrm{cm}$ and hence the gating circuit has to operate at excessive speeds, e.g., picoseconds. Further, due to breathing the skin may move by more than a few centimeters, making it impossible to set a gating threshold that captures the implant's signal yet rejects skin reflections.
} 
operate a local high-frequency clock (with frequency $f_{c}$ ) on the backscatter device. Thus, if the incident signal has frequency $f$, the backscatter device uses the local clock to generate frequency $f_{c}$, and RF mixers to mix these two frequencies and generate $f+$ $f_{c}$ or $f-f_{c}$. This is usually done to shift existing Wi-Fi signals to adjacent Wi-Fi frequency bands to avoid interference. This approach works well for IoT devices equipped with batteries to run the high-frequency (tens of $\mathrm{MHz}$ ) clock, and with no size-restrictions. However, their battery requirement, size and complexity make them undesirable for in-body use.

So, the question becomes how we can shift frequencies without using any active wireless components. Our insight is to leverage non-ideal circuit behavior to generate frequency shifts. In traditional RF design, circuits are designed to exhibit linear behavior, i.e., if a circuit (like an RF amplifier) receives an input signal $s$, it's output defined by $f_{\text {linear }}(s)$ is given by:

$$
f_{\text {linear }}(s)=\gamma s
$$

where $\gamma$ is a complex number. Thus, the system just scales signal magnitude and adds a constant phase to the signal.

In contrast to linear systems which apply a linear transformation to the input signal, non-linear systems can apply generally polynomial transforms to the input signal. Specifically, for an input signal $s$, the output signal $f_{\text {non-linear }}(s)$ is given by:

$$
f_{\text {non-linear }}(s)=\gamma_{0} s+\gamma_{1} s^{2}+\gamma_{2} s^{3}+\ldots
$$

where $\gamma_{0}, \gamma_{1}, \ldots$ are complex numbers. To understand what this non-linear behavior means for the frequencies contained in a signal, consider a signal $s$ that is a sum of two sine waves of different frequencies $f_{1}$ and $f_{2}$, i.e. $s=\sin \left(2 \pi f_{1} t\right)+\sin \left(2 \pi f_{2} t\right)$, where $t$ is the time.

$$
\begin{aligned}
& \gamma_{1} s^{2}=\gamma_{1}\left(\sin \left(2 \pi f_{1} t\right)+\sin \left(2 \pi f_{2} t\right)\right)^{2} \\
& =\gamma_{1}\left(\sin ^{2}\left(2 \pi f_{1} t\right)+\sin ^{2}\left(2 \pi f_{2} t\right)+2 \sin \left(2 \pi f_{1} t\right) \sin \left(2 \pi f_{2} t\right)\right) \\
& =\frac{\gamma_{1}}{2}\left(\left(2-\cos \left(2 \pi 2 f_{1} t\right)-\cos \left(2 \pi 2 f_{2} t\right)\right.\right. \\
& \left.+\cos \left(2 \pi\left(f_{1}-f_{2}\right) t\right)-\cos \left(2 \pi\left(f_{1}+f_{2}\right) t\right)\right)
\end{aligned}
$$

Here the last equation is derived using standard trigonometric formulae. The final step in equation 8 shows that the output signal contains different linear combinations of the input frequencies, i.e., $2 f_{1}, 2 f_{2}, f_{1}-f_{2}$ and $f_{1}+f_{2}$. Each of these frequency components are referred to as harmonics. Similarly, it can be shown that the third order term contains third order combinations like $3 f_{1}, 3 f_{2}, 2 f_{1}+f_{2}, 2 f_{2}+f_{1}, 2 f_{2}-f 1, \ldots$

Note that, this behavior is typically non-ideal in circuits and RF designers try to minimize non-linear behavior. However, in the context of in-body backscatter, this presents us with an opportunity to shift the signal in frequency using a non-ideal RF component. This allows us to transmit a signal composed of two sine waves at different frequencies $f_{1}$ and $f_{2}$. When the in-body implant receives the signal, it uses the non-linear behavior to create signals at frequencies which are combinations of the twoincident frequencies. While the human body reflects signals at just $f_{1}$ and $f_{2}$, the signal reflected by the implant contains signals at frequencies $f_{1}+f_{2}, f_{1}-f_{2}, 2 f_{1}-f_{2}$, and so on. This allows us to filter out reflected signals from the human body at $f_{1}$ and $f_{2}$ and isolate the signal reflected by the in-body device.

Finally, how can we produce non-linearities using simple passive components, i.e., components with no battery? As we said before, most RF-circuits are designed to exhibit linear behavior. Thus, to produce non-linearities, we turn to a fundamental non-linear component, the diode. A diode is the simplest non-linearity producing component, that is completely passive and is typically used as building block in larger, more complex circuits. By using a diode connected to an antenna, we can design a simple RF front-end for the implant. It can receive signals at two frequencies, $f_{1}$ and $f_{2}$, and backscatter combinations of these frequencies, thereby allowing a receiver outside the body to isolate the backscattered signal.

\subsection{System Design}

We use the design shown in the inlet in Fig. 3, which is similar to standard passive RFIDs except for the nonlinear diode connecting the antenna to the rest of the system. Given an input signal, the diode mixes the frequencies in the signal creating second and third order frequencies. These frequencies are fed to a switch, which modulates the signal. Specifically, the device can turn the switch on or off to communicate its data using on-off keying, as in passive RFIDs. The backscatter signal can be demodulated by the receiver using standard demodulation techniques. Note that, even for high data applications like smart capsules which take images of the GI tract, the data requirements are few $100 \mathrm{kbps}$ since they typically transmit one or two small frames per second [3]. Thus, a simple encoding like onoff keying suffices for communication. Notice that our design is completely passive since a diode is a passive component that does not need to be powered. Furthermore, a diode is a very small and simple component that can be added to an in-body transceiver with negligible size change.

Frequency Selection: One important system design consideration for in-body backscatter is the choice of frequencies to be transmitted. There are two constraints that must be met.

- Safety Limits:The safety standards for in-body transmissions need to be met. As past work has shown [2], it is safe to transmit up to $28 \mathrm{dBm}$ for an on-body antenna at frequencies around $1 \mathrm{GHz}$. This power level suffices for ReMix. Our system experiences a loss of around 80 to $100 \mathrm{~dB}$ in the human body at frequencies around $1 \mathrm{GHz}$. But to be on the conservative side, even if one assumes the loss is up to $130 \mathrm{~dB}$, the expected received signal strength is $\approx-100$ $\mathrm{dBm}$. This low power can support $1 \mathrm{MHz}$ wide on-off keying transmissions using standard receiver design $[11,55]$.

- FCC Regulations: The FCC regulates which frequencies can be used - specifically it has set aside several bands around 1 


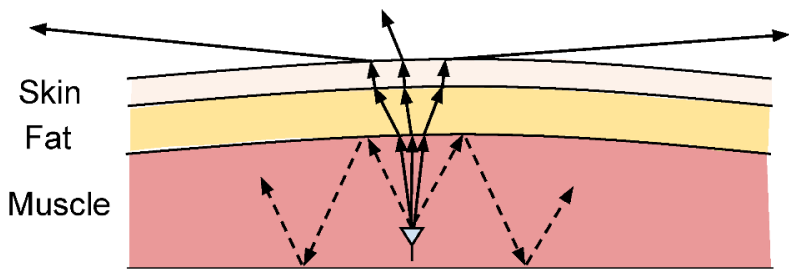

Figure 4-Due to high $\epsilon_{r}$ for muscle, only the signal travelling along a small angle can exit the body. All other signals get reflected.

GHz (174 MHz-216 MHz, 470 MHz-668 MHz, $1395 \mathrm{MHz}-$ $1400 \mathrm{MHz}, 1427 \mathrm{MHz}-1432 \mathrm{MHz}$ ) for biomedical telemetry services, in addition to the ISM bands (FCC 15.241, 15.242, part 95, subpart $\mathrm{H}$ ([18])). ReMix transmitters can use any two frequencies in this range. For example, one can transmit at $570 \mathrm{MHz}\left(f_{1}\right)$ in the biomedical telemetry band and $920 \mathrm{MHz}\left(f_{2}\right)$ in the ISM band. The backscattered signals can then, for example, be received at $1490 \mathrm{MHz}\left(f_{1}+f_{2}\right)$ and $1270 \mathrm{MHz}\left(2 f_{2}-f_{1}\right)$. The frequencies transmitted by the backscatter device inside the body do not violate FCC regulations because their power levels are much lower than FCC spurious transmission limits ([27]). In part 15.209, FCC limits the effective radiated power of spurious transmissions (like harmonics) of unlicensed devices to be less than -52 $\mathrm{dBm}$ (for frequency bands over $100 \mathrm{MHz}$ ), which is much larger than our backscattered power.

\section{IN-BODY BACKSCATTER LOCALIZATION}

We discuss the key challenges in positioning in-body devices using RF signals and how ReMix overcomes them. While we explain our insights in the context of backscatter, they naturally apply to non-backscatter RF signals.

\subsection{Understanding the Positioning Challenge}

State-of-the-art RF localization systems [33, 60, 61, 63] operate in two steps. In the first step, they use the phase of the wireless channel between the transmitter and the receiver to measure the angle-of-arrival of the signal or distance between the transmitter and the receiver. In the second step, they assume the path travelled by the signal is straight, and apply basic geometry to locate the transmitter. For in-body RF signals, both these steps are bound to fail if applied as is.

First, let us consider the phase of the wireless channel. As shown in Eq. 1, the phase in air or vacuum, $\phi$, is given by: $\phi=-2 \pi f \frac{d}{c} \bmod 2 \pi$. Here, $f$ is the frequency of the signal and $c$ is the speed of EM waves in vacuum. Hence, the phase of the wireless channel linearly depends on the distance travelled by the signal. In contrast, and as discussed in $\$ 3$, the phase accumulated by the signal in a biomaterial is scaled by $\alpha=$ $\operatorname{Re}\left(\sqrt{\epsilon_{r}}\right)$, where $\epsilon_{r}$ is the electrical permittivity of the material. Thus, when the signal traverses the body, its phase is:

$$
\phi=-2 \pi \frac{f}{c}\left(\sum_{i} \alpha_{i} d_{i}\right) \bmod 2 \pi
$$

where the sum is over the various materials traversed by the signal (air, skin, fat, muscle, etc.), $\alpha_{i}$ is the real square-root of the electrical permittivity of material $i$, and $d_{i}$ is the distance traveled in that material. This means that when the signal traverses multiple materials, the phase is no longer a simple function of the distance between the transmitter and the receiver.

Second, even if we could measure the distance traversed or angle-of-arrival of the signal, how does one map it to a location? As discussed in $\S 3$, the signal experiences refraction (bending) at the interface between different material (e.g., the interface between air and skin). Thus, the assumption that the signal travels between two points along straight lines no longer holds. As a result, the geometric model of intersecting distances or angles from multiple viewpoints doesn't apply.

Third, we need to take into account that our signal travels the entire distance at different frequencies. The signal from the transmitter to the backscatter device consists of two frequencies $\left(f_{1}\right.$ and $f_{2}$ ), while the signal measured at the receiver after being backscattered is at $f_{1}+f_{2}, 2 f_{1}+f_{2}$, and other frequency combinations. Together, these three factors are the main challenges facing in-body localization.

\subsection{Principles of Positioning}

Before we introduce ReMix's positioning algorithm, we discuss a few key insights that we build our algorithm on.

(a) RF signals exit the body from a small region on the surface: In $\S 3(\mathrm{e})$, we made the observation that it does not matter how the signal arrives from air, it enters the body only close to the direction of the normal on the surface. Since RF propagation is reversible, this means that it is also not possible for the RF signal to escape from the body through all possible directions. In fact, it can escape only from a small region around the normal on the surface, as shown in Fig. 4. The reason is the property of refraction. Specifically, when an RF signal travels from a high permittivity material (like human body) to a low permittivity material (like air), it bends away from the direction perpendicular to the interface between the materials. Substituting the electrical permittivity values for body tissues in Eq. 5 shows that the cone in Fig. 4 is about $8^{\circ}$. In-body signals that arrive more than $8-10^{\circ}$ away from the normal on the surface typically reflect internally and do not escape to the air.

(b) No in-body multipath: As a corollary of the first point, inbody multipath either does not exist or is very weak compared to the direct path. Any signal that is reflected back into the body has to traverse multiple $\mathrm{cm}$ of human tissue and face multiple reflections before it can escape the human body. Because of the exponential attenuation caused by human tissue, this signal will be very low power compared to the direct path emanating out of the body. This is quite in contrast to large scale in-air localization systems where the line-of-sight path can be much weaker than multipath because of obstructions. 
(c) Material order and interleaving can be ignored: The human body has multiple layers of tissues interleaved with each other. For example, skin and muscle are alike in electrical properties but are separated by fat which is closer to air. Further, the same material can appear in multiple layers (e.g., air-skinfat-muscle-fat-msucle). This complex layering structure makes it challenging to model refraction at various interfaces. We can prove, however, that for parallel layers, order and interleaving can be changed with no impact on the total phase of the signal. ${ }^{2}$ Since human tissues tend to be layered on top of each other, the assumption of parallelism is a reasonable approximation. We prove this observation in the appendix. We also verify this property empirically using multiple layers of pork belly in $\S 10$. This observation implies that the multiple layers of the human body can be rearranged for modeling and approximated to be grouped in two major layers: one layer comprising oil based tissues (like fat) and another layer comprising water based tissues (like skin and muscle).

\section{ReMix's Localization Algorithm}

We introduce a localization algorithm that is particularly customized for in-body RF signals. The algorithm works in two steps. First, it estimates the distances traveled by the signal as if it were traveling in air. We call such values the effectivein-air distances. Second, it models signal paths with linear splines (piecewise linear segments). The length of each segment refers to the stretch of the path in a particular material (air, fat, muscles). It then solves an optimization problem that maps the effective distances to the correct splines that match the actual paths traveled by the signal. (For simplicity, all phase equations are expressed ignoring the initial difference in oscillator phase between transmitter and receiver which can be measured during the calibration phase.)

\subsection{Measuring Effective In-Air Distances}

Consider a signal traveling from a transmitter to a receiver through $L$ different biomaterials. Assume that it travels distance $d_{i}$ in biomaterial $i$, with phase scaling factor $\alpha_{i}=\operatorname{Re}\left(\sqrt{\epsilon_{r i}}\right)$. We define effective in-air distance, $d_{\text {eff }}$, traveled by the signal as:

$$
d_{e f f}=\sum_{i=0}^{N-1} \alpha_{i} d_{i}
$$

Combining Eq. 10 with Eq. 9, the phase, $\phi$, of the signal observed by the receiver is:

$$
\phi=-\frac{2 \pi f d_{e f f}}{c} \bmod 2 \pi .
$$

Thus, an alternative definition for the effective in air distance is that, if travelled in air, it would result in the received phase.

So, how do we compute the effective distances? Recall that ReMix has two transmit antennas that transmit $f_{1}$ and $f_{2}$, and

\footnotetext{
${ }^{2}$ Reordering of layers affects the amplitude due to more reflections.
}

a number of receive antennas. Let $d_{1}$ and $d_{2}$ be the effective distances from the two transmitters to the backscatter device, and $d_{r}$ the effective distance from the backscatter device to receiver $r$. The transmitters are transmitting frequencies $f_{1}$ and $f_{2}$, while the receivers receive the non-linear mixing of these two signals at frequencies $f_{1}+f_{2}, 2 f_{1}-f_{2}$, and other linear combinations. Let us consider the phase of $f_{1}+f_{2}$ measured at receive antenna $r$, which can be given by:

$$
\phi_{i}=-\frac{2 \pi}{c}\left(f_{1} d_{1}+f_{2} d_{2}+\left(f_{1}+f_{2}\right) d_{r}\right) \bmod 2 \pi
$$

This phase equation is a combination of three components. The first two components correspond to the phase of the signal from the transmit antenna to the device. They combine based on the particular non-linear component of the signal that we receive. Since, we are considering just the non linear component $f_{1}+f_{2}$, which is just the sum of the frequencies, the corresponding phases also add up (this follows directly from Eq. 8). Eq. 12 gives us one equation in terms of three unknowns, $d_{1}, d_{2}$, and $d_{r}$. We need more equations to solve for these unknowns. Note, now, that the non-linearity generates various frequency mixes, which provide additional equations. For example, we can write a similar equation for $2 f_{1}-f_{2}$. The phase, $\psi_{i}$, measured at this frequency is given by:

$$
\psi_{i}=-\frac{2 \pi}{c}\left(2 f_{1} d_{1}-f_{2} d_{2}+\left(2 f_{1}-f_{2}\right) d_{r}\right) \bmod 2 \pi
$$

Once again, note that the phase accumulated by the signal combines in the same way as the frequencies.

To simplify Eq. 12 and Eq. 13, we combine them as:

$$
\begin{aligned}
\phi_{i}+\psi_{i} & =-\frac{2 \pi}{c} 3 f_{1}\left(d_{1}+d_{r}\right) \quad \bmod 2 \pi \\
2 \phi_{i}-\psi_{i} & =-\frac{2 \pi}{c} 3 f_{2}\left(d_{2}+d_{r}\right) \quad \bmod 2 \pi
\end{aligned}
$$

Thus, we get equations expressed as summed distances from each of the transmitters to the receivers. At this point, we cannot use more harmonics to solve for individual distances since they will just yield equations that are linearly dependent on these two harmonics. However, we can use another receiver $r^{\prime}$ to get two additional equations that are functions of $d_{1}, d_{2}$ and $d_{r^{\prime}}$. Thus, given at least two receive antennas, these four equations can be solved to obtain $d_{1}, d_{2}, d_{r}$ and $d_{r^{\prime}}$. More antennas can be used to improve accuracy of distance estimates. ${ }^{3}$

\subsection{Mapping Effective Distance to Actual Location}

Now that we have the effective in-air distances between the in-body backscatter device and the transmit and receive antennas outside the body, we want to map those effective distances to the actual physical location of the backscatter

\footnotetext{
${ }^{3}$ We note that all phase equations are $\bmod 2 \pi$. To resolve ambiguity due to the phase wrapping around, ReMix, like past work [60], uses a small frequency band around each of the transmitted frequencies - i.e., instead of just transmitting $f_{1}$ and $f_{2}$, ReMix sweeps through its transmission in a small band of 10 $\mathrm{MHz}$ around $f_{1}$ and $f_{2}$.
} 


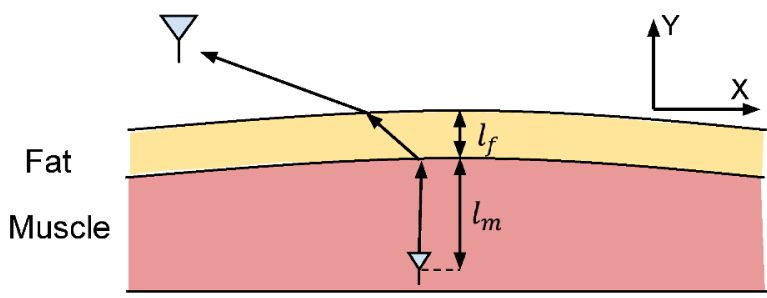

Figure 5-Localization Model: The signal from the in-body device travels through two layers (fat, muscle) to reach ReMix antennas.

device. At this stage we can drop the distinction between the transmit and receive antennas and treat all effective distances in the same way.

As we said before, the in-air effective distance does not translate into physical distances directly. Furthermore, since the signals do not travel in a straight line, the distances cannot simply be intersected from the different transmitters and receivers to get the right location. To solve this problem, we model signal propagation inside the human body as linear splines (instead of a straight line). Thus, propagation in each layer is linear, but across layers, it can change directions.

Model: As discussed before, human tissues can be classified as either oil (like fat) or water based (like muscle). Furthermore, different layers can be rearranged such that the muscle-based tissues occur together and the fat-based tissues occur together. Thus, we model the human body as a two-layer system, as shown in Fig. 5. For ease of exposition and visualization, we discuss the algorithm in the 2D XY plane. An extension to 3D is straightforward. The in-body backscatter implant is located at $X$, where $X$ is a tuple of its $(x, y)$ coordinates. The implant is covered by a layer of muscle with depth $l_{m}$ (relative permittivity $\epsilon_{r m}$ ). Then, there is a layer of fat, which has depth $l_{f}$ (relative permittivity $\epsilon_{r f}$ ). Thus, our model, $\mathcal{M}$ has three latent variables $\left(X, l_{m}, l_{f}\right)$. In addition to these latent variables, the model has fixed parameters, $\Theta$ : the position of each antenna, $X_{i}$ for $i=1, \ldots, N$, and the permittivity of biomaterials $\epsilon_{r f}$ and $\epsilon_{r m}$. The observations made by the model are the effective distance measurements, $d_{i}$, from the implant to each of the antennas. Then, the goal of the model is to estimate the hidden variable $\left(X, l_{m}, l_{f}\right)$ given a set of observations.

Constraints: Next, how does the model constrain the structure of the splines for each path? Let us consider the effective inair distance $d_{i}$ measured at the $i^{\text {th }}$ antenna. In our model, the effective in-air distance is modeled by a spline comprised of 3 different segments: an in-air segment of length $d_{a}^{i}$, in-fat segment of length $d_{f}^{i}$ and in-muscle segment, $d_{m}^{i}$. Together, when these physical distances are scaled by their respective scaling factors and summed together, they should yield the effective-in-air distance $d_{i}$. The estimation of the individual segments of these splines is governed by two sets of constraints:
- Refraction Constraints: Let us say the angle of incidence inside fat, muscle and air is $\theta_{f}^{i}, \theta_{m}^{i}, \theta_{a}^{i}$ respectively. Then:

$$
\begin{gathered}
\operatorname{Re}\left(\sqrt{\epsilon_{r a}}\right) \sin \theta_{a}^{i}=\operatorname{Re}\left(\sqrt{\epsilon_{r f}}\right) \sin \theta_{f}^{i} \\
\operatorname{Re}\left(\sqrt{\epsilon_{r m}}\right) \sin \theta_{m}^{i}=\operatorname{Re}\left(\sqrt{\epsilon_{r f}}\right) \sin \theta_{f}^{i}
\end{gathered}
$$

- Geometric Constraints: If $\left(X_{i}-X\right)_{1}$ denotes the horizontal dimension of the difference between two positions, then:

$$
\begin{array}{r}
d_{a}^{i}=\frac{l_{a}}{\cos \theta_{a}^{i}}, d_{f}^{i}=\frac{l_{f}}{\cos \theta_{f}^{i}}, d_{m}^{i}=\frac{l_{m}}{\cos \theta_{m}^{i}} \\
d_{a}^{i} \sin \theta_{a}^{i}+d_{f}^{i} \sin \theta_{f}^{i}+d_{m}^{i} \sin \theta_{m}^{i}=\left(X_{i}-X\right)_{1}
\end{array}
$$

where $l_{a}$ is the depth of air which is equal to the total distance along the vertical dimension minus the depth of muscle and fat combined.

We now have a system with 6 variables $\left(d_{a}^{i}, d_{m}^{i}, d_{f}^{i}, \theta_{m}^{i}, \theta_{f}^{i}, \theta_{a}^{i}\right)$ and 6 independent equations (Eq. 15 and 16). This is solvable numerically using ray tracing methods. Finally, $d_{a}^{i}, d_{f}^{i}$ and $d_{m}^{i}$, thus obtained are functions of the latent variables $\left(X, l_{m}, r_{f}\right)$. Thus, we denote the length of the segments of the spline corresponding to antenna $i$ in air, fat and muscle by $d_{a}^{i}\left(X, l_{m}, l_{f}\right), d_{f}^{i}\left(X, l_{m}, l_{f}\right), d_{m}^{i}\left(X, l_{m}, l_{f}\right)$ respectively.

Optimization: Now that we modeled the individual segments of the splines as functions of the latent variables in the model, we want to leverage the observed effective in-air distances to estimate the latent variables. Specifically, for each antenna, we minimize the L2-norm of the observed effective in-air distances $\left(d_{i}\right)$ and the distance obtained by the scaled sum of the spline segments $\left(d_{a}^{i}()+.\alpha_{f} d_{f}^{i}()+.\alpha_{m} d_{m}^{i}().\right)$. As before, $\alpha=\operatorname{Re}\left(\sqrt{\epsilon_{r}}\right)$. Thus, combining distance measurements from multiple antennas, our optimization function can be written as:

$$
\hat{X}, \hat{l_{m}}, \hat{l_{f}}=\arg \min _{X, l_{m}, l_{f}} \sum_{i=1}^{N}\left\|d_{a}^{i}(.)+\alpha_{f} d_{f}^{i}(.)+\alpha_{m} d_{m}^{i}(.)-d_{i}\right\|^{2}
$$

where $\hat{X}, \hat{l_{m}}, \hat{l_{f}}$ are the optimal values of the latent variables. This optimization problem is convex in each of the hidden variables $\left(X, l_{a}, l_{f}\right)$ for $\epsilon_{r}$ value ranges of human tissues. Furthermore, it has one local maximum. It can be framed as a standard convex optimization problem and solved using convex optimization techniques. By doing so, this optimization can accurately estimate the position of the device by modeling the spline structure.

\section{IMPLEMENTATION}

ReMix's implementation has two primary components: the in-body backscatter device and the out-of-body ReMix transceiver that generates the two frequencies and listens for the backscatter signal. For our implementation of the in-body backscatter device, we use a Schottky detector diode from Skyworks Solutions [52]. The size of the diode is $1.6 \mathrm{~mm}$ by $0.8 \mathrm{~mm}$. We 


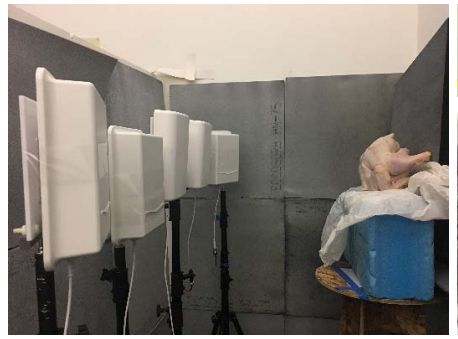

(a) System Setup

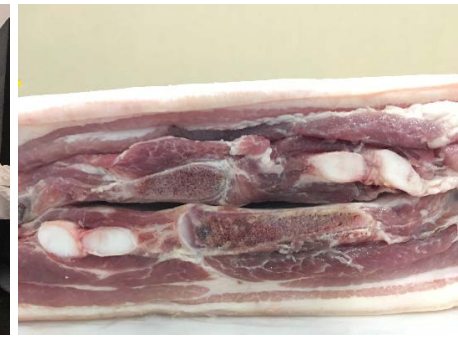

(b) Pork Belly

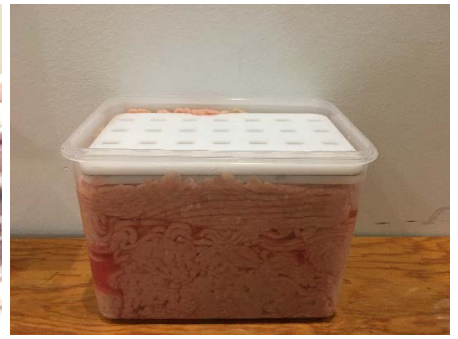

(c) Localization Setup

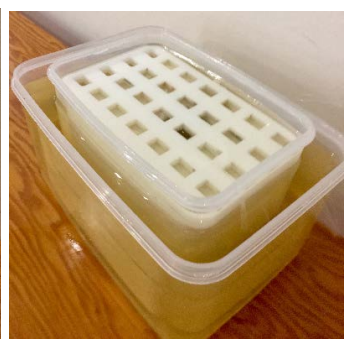

(d) Phantom Tissue

Figure 6-ReMix Implementation:ReMix's system setup is shown in (a). To emulate human tissue behavior, we conduct experiments with animal tissues like (a) whole chicken, (b) pork belly, and (c) ground chicken in addition to (d) human tissue phantoms. In (d), the outer layer is fat phantom and the inner box is filled with muscle phantom.

connect this diode to the PC30 dipole antenna from Taoglas [56]. We note that this antenna is $7.5 \mathrm{~cm}$ long and its gain is around $0 \mathrm{~dB}$ in-air for the band of interest. However, its an off-the-shelf antenna optimized for in-air transmissions and its design is not optimized for in-body transmissions. Smaller antennas the size $([19,38,43])$ of a grain of rice have been used in in-body RFIDs and one could leverage these designs for better performance. However, the exploration of antenna design is out of the scope of this paper.

For out-of-body transceiver, we use two patch antennas for transmissions and three patch antennas for reception. We use this set of five antennas for localization. For communication, a single receive antenna is sufficient. A picture of this setup is shown in Fig. 6(a). To avoid mixing of the two transmitted frequencies in the transmission circuit, we use separate transmit chains for both the transmitted frequencies. The antennas are typically placed from $50 \mathrm{~cm}$ to $2 \mathrm{~m}$ away from the subject for our experiments. These antennas are connected to USRP software radios ([17]). The transmit frequencies used are 830 $\mathrm{MHz}\left(f_{1}\right)$ and $870 \mathrm{MHz}\left(f_{2}\right)$. We use two harmonics $910 \mathrm{MHz}$ $\left(2 f_{2}-f_{1}\right)$ and $1700 \mathrm{MHz}\left(f_{1}+f_{2}\right)$. Our choice of frequencies is illustrative and another set of frequencies can be chosen as discussed in $\S 5$. Our choice was governed by the availability of off-the-shelf hardware in these bands.

The signal transmission and reception were done using USRP X300 software radios and UBX daughterboards. The USRPs were synchronized using an external $10 \mathrm{MHz}$ clock. The USRPs were programmed using the UHD library in C. The signals received from the USRPs were collected on a desktop PC over ethernet and the received signals were processed in Matlab.

\section{Human Tissue Emulation}

We emulate human tissues using two methods commonly used in the literature on in-body imaging and power transfer [2, 13, 44, 49, 51]. First, we use animal tissues like whole chicken (Fig. 6(a)), ground chicken meat (Fig. 6(c)) and pork belly (Fig. 6(b)). These tissues have complex layer structures and contain skin, muscle, fat, bones, etc. Further, the EM properties of these tissues is similar to human tissues [26, 53]. Thus, they provide an ideal environment for emulating human tissues.
Second, we use phantom tissues have been proven to exhibit electrical properties similar to human tissues in frequencies up to $2500 \mathrm{MHz}[1,28,36,46]$. These phantoms can emulate both water-based (muscle) and oil-based (fat) tissues. The muscle phantoms are made using Polyethylene powder and Agarose [28], and the fat phantoms are made mainly of vegetable oils and gelatin [36]. We vary the amount of fat and muscle to emulate human tissues with more fat or more muscle.

An in-body implant placed inside chicken tissue or human phantom is not visible to the naked eye. Thus, for localization experiments where precise ground truth location is required, we use the setup shown in Fig. 6(c). The ground chicken meat or human phantom is packed in a plastic container and covered with a lid. The lid has slits cut into it using precise (sub-millimeter) laser cutting methods. The implant is placed through the slits at a fixed depth below the surface. By varying the slit that the implant is inserted into, the implant's location in the human phantom or chicken tissue can be changed.

\section{EMPIRICAL RESULTS}

We discuss our evaluation of ReMix below.

\subsection{Microbenchmarks}

Non-linear Behavior of Diode: ReMix exploits the non-linear behavior of a diode for isolating the in-body backscatter signal. A mathematical explanation of the non-linear behavior is given in $§ 5$. To understand the practical manifestation of the nonlinear behavior, we design a simple experiment. A diode is connected to an antenna and placed in the air. Two transmitters, each transmitting a single frequency signal, are placed at a distance of $1 \mathrm{~m}$ from the diode-antenna system. A receive antenna is placed $1 \mathrm{~m}$ from the diode on the same side as the transmitter. The power observed by this receiver on different frequencies is plotted in Fig. 7(a).

As can be seen in the figure, the diode produces multiple non-linear combinations of the input signal. The second order harmonics $\left(2 f_{1}, 2 f_{2}, f_{1}+f_{2}\right)$ are lower in power than the main signal peaks, but higher in power than the third order harmonics $\left(2 f_{1}-f_{2}, 3 f_{1}\right.$, etc.). The variation in power in different frequency 


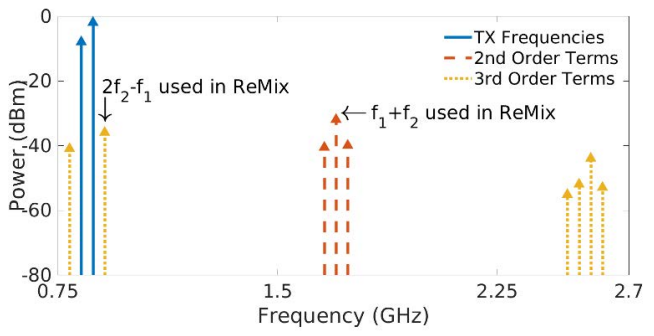

(a)

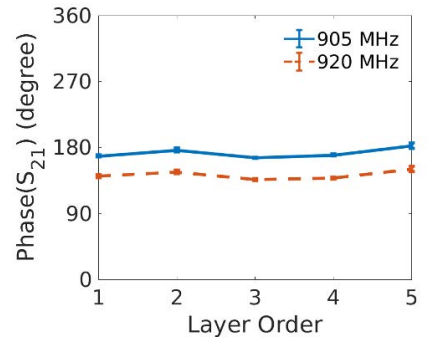

(b)

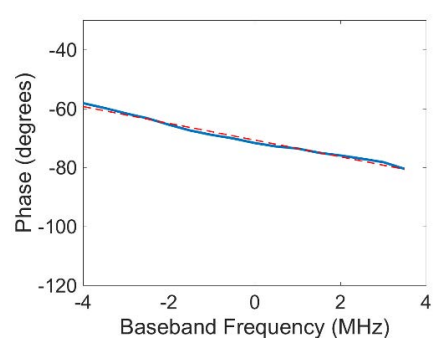

(c)

Figure 7-ReMix Microbenchmarks:(a) ReMix's design produces non-linear mixing of transmitted frequencies which can be used to filter out strong human body reflections, (b) Interchange of layers (Tab. 1) does not impact signal phase, (c) Linear relationship between phase (in blue) and frequency shows non-existent or mild multipath in human body.

\begin{tabular}{|c|l|}
\hline Config & Layer Structure \\
\hline 1 & Skin, Fat, Muscle, Fat, Muscle, Muscle, Bone \\
\hline 2 & Muscle, Fat, Muscle, Fat, Skin, Muscle, Bone \\
\hline 3 & Skin, Fat, Muscle, Fat, Muscle, Bone, Muscle \\
\hline 4 & Muscle, Fat, Muscle, Fat, Skin, Bone, Muscle \\
\hline 5 & Bone, Muscle, Skin, Fat, Muscle, Fat, Muscle \\
\hline
\end{tabular}

Table 1-Configurations for Layer Interchange Experiment

bands is caused by: (a) the diode conversion loss, i.e., different harmonics have different power after the non-linearity of the diode is factored in, (b) the propagation loss over air - since different frequencies have different loss over air, the observed power is higher for lower frequencies.

This experiment indicates that a diode's non-linear behavior produces reflected signal at multiple frequency bands. ReMix uses these signals for in-body backscatter.

Interchange Layers: In $\S 6.2$, we observed that the order of layers can be changed without impacting the phase of the signal. In order to validate this observation, we use a big chunk of pork belly. Pork belly is composed of layers of fat, muscle and bones. A big chunk of meat is placed below the transmit antenna so as to avoid any leakage over the air. Then, the transmit antenna is covered by different sequences of multiple layers given in Tab. 1. Finally, a receive antenna is placed on top of this setup, about $10 \mathrm{~cm}$ away. Each configuration of the layers given in Tab. 1 is repeated 5 times. The signal phase is measured at the receive antenna at two different frequencies.

The phase observed on the receive antenna and its standard deviation are plotted in Fig. 7(b). As seen in the figure, the phase remains almost constant across these measurements. The standard deviation in these measurements is 8 degrees which is within quite small and within the measurement errors. This shows that re-ordering different tissue layers in-body do not impact the phase of the received signal.

Lack of in-body Multipath: In 6.2, we discussed why the direct path for a signal from inside the body is much stronger than any reflected paths. While mapping the multipath directly would either need a large antenna array or a large frequency bandwidth, we use an approximate method to verify this assumption. Specifically, for a fixed distance between transmitter and receiver, signal phase changes linearly with frequency if there is no multipath. When there is significant multipath, the phase is no longer linear. Thus, to verify whether this observation holds, we put ReMix's backscatter device inside a box full of chicken meat and observe the phase of the reflected signal across a $8 \mathrm{MHz}$ frequency band. Each of the transmitter frequencies is moved by up by $8 \mathrm{MHz}$ in steps of $0.5 \mathrm{MHz}$ and the received signal phase is observed and plotted in Fig. 7(c). As can be seen in this figure, the phase has a linear relationship with distance. This indicates that the in-body multipath for ReMix is mild to non-existent.

\subsection{Backscatter Communication}

To evaluate the backscatter communication capabilities of ReMix, we use three different setups: ground chicken meat, human phantom tissues and whole (dead) chicken. The human phantom is designed to have $1.5 \mathrm{~cm}$ fat, followed by muscle. In each of these setups, we measured the Signal to Noise ratio (SNR) for depths up to $8 \mathrm{~cm}$ of tissue. Typically, human abdomen muscle can be as deep as $1.6 \mathrm{~cm}$ and the small intestine is around $1 \mathrm{~cm}$ deep [16]. Since muscle and intestine have high attenuation for RF signals, our tests including 7-8 cm muscle should present an insight into the worst case behavior of ReMix's communication capabilities. Finally, we operate the system at a $1 \mathrm{MHz}$ bandwidth for all these measurements.

We plot the SNR observed at a single harmonic in Fig. 8. As can be seen in the figure, the SNR of the signal decreases as the depth in tissue increases. The average SNR obtained in chicken and phantom are $15.2 \mathrm{~dB}$ and $16.5 \mathrm{~dB}$ respectively. Furthermore, even at depths of $8 \mathrm{~cm}$, a depth which is larger than human muscle depth, the SNR is as high as 7 to $11 \mathrm{~dB}$. Notice that the human phantom and the chicken meat exhibit similar behavior. This is along expected lines since they have similar dielectric properties.

Finally, while we cannot make precise measurements and incisions in whole dead chicken, we measure the SNR for 5 random locations inside the chicken body. The mean SNR observed was around $23 d B$. This is higher than ground chicken or human phantom because the muscle thickness in the whole 


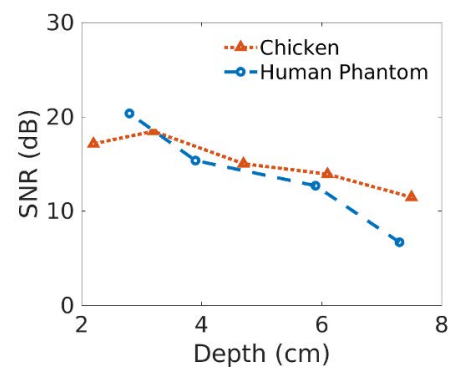

Figure 8-ReMixBackscatter:ReMix can achieve an average SNR of over $15 \mathrm{~dB}$ for $1 \mathrm{MHz}$ frequency band even when the tissue depth is up to $8 \mathrm{~cm}$ (human muscle depth is 2-3 cm [16]).This SNR can be improved by combining multiple antennas.

chicken is just $2-5 \mathrm{~cm}$, which is lower than the muscle thickness we analyzed for ground chicken and human phantom.

Combining Across Antennas: The SNR reported above is the SNR for a single antenna. Since ReMix has multiple receive antennas, it can combine the signal across these antennas using techniques like maximal ratio combining (MRC) [57] to achieve higher SNR. Fig. 8 plots the SNR after combining the signal received at our 3 receive antennas using MRC. As expected, the combination gives us an average gain of $5-6$ $\mathrm{dB}$ with 3 antennas. The performance is similar for both, the chicken tissue and the human phantom. This implies that we can use the multiple antennas to improve SNR in-body.

Data rates: ReMix uses On-off keying (OOK) for its data transmission. OOK is robust to noise and can achieve a datarate of $1 M b p s$ with a bit error rate of $10^{-4}$ at SNR of around 12 $\mathrm{dBm}$ and bit error rate of $10^{-5}$ at SNR of around $14 \mathrm{dBm}$ $[11,55]$. In realistic cases (muscle depth $<5 \mathrm{~cm}$ ), the SNR of ReMix is in the $12-20 \mathrm{~dB}$ range even with a single receive antenna. Thus, ReMix can provide efficient communication for in-body devices like a wireless capsule endoscope, which have a requirement of few hundreds of kbps.

\subsection{In-Body Localization}

We evaluate ReMix's localization accuracy using two different arrangements. First, we use chicken meat, covered with fixed size slits in a plastic cover (shown in Fig. 6(c)). The slits are 1 inch apart along each dimension. In the second setup, we use human phantoms. The human phantom tissues are designed to emulate layers of fat and muscle $a$ la the human body. As shown in Fig. 6(d), the muscle phantom is placed inside the smaller box covered with slits spaced 1 inch apart along each dimension. The muscle phantom is surrounded by a layer of fat. The thickness of the fat layer is varied between $1-3 \mathrm{~cm}$ randomly to emulate variation in body structure. The goal of this setup is to be true to human tissue layers.

We measure localization error of the backscatter device placed through the slits in the cover. We make 50 different measurements in each of the two setups, and report the CDF of localization error in Fig. 10(a). As the figure shows, the median localization error for ReMix is $1.4 \mathrm{~cm}$ in chicken tissue and $1.27 \mathrm{~cm}$ in human phantom, and the maximum is $2.2 \mathrm{~cm}$

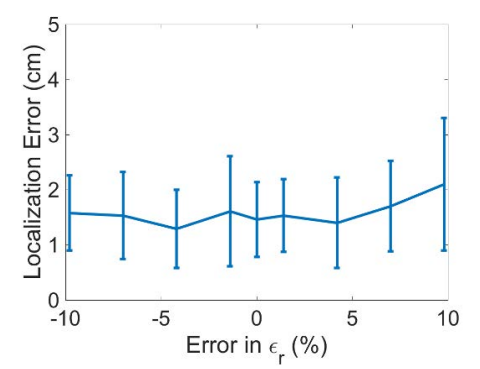

Figure 9- $\epsilon_{r}$ Variance: For typical variance in human tissue properties, ReMix's localization error continues to be $<2 \mathrm{~cm}$.

in chicken tissue and $1.8 \mathrm{~cm}$ in human phantom tissue. Note that, this error is much lower than the $5 \mathrm{~cm}$ accuracy required for depositing biomarkers in the colon for wireless capsule endoscopy [49] and can enable this application. Furthermore, this error is $2 \mathrm{X}$ lower than the theoretical lower bound on RSS based in-body localization achievable with 32 antennas [64].

To delve deeper into the results, we discuss two aspects of the localization. First, we study how the use of refraction model impacts the localization error. Thus, we use ReMix's distance based model without the refraction model and observe the error along two axis: lateral localization error along the surface of the body and error in measuring the depth of the device in the body. The CDF of the surface error and depth error are shown in Fig. 10(b). The median surface and depth error for ReMix are $1.04 \mathrm{~cm}$ and $0.75 \mathrm{~cm}$ respectively. Without ReMix's refraction model, however, the error increases. The surface error and depth errors in that case are $3.4 \mathrm{~cm}$ and $6.1 \mathrm{~cm}$ respectively. Note that, without the refraction model, the error in estimating depth of the in-body device is higher than the error in estimating position along the surface. A simple way to understand is to compare this with refraction in visible light. When one observes a coin placed in water, it appears at the same lateral position, but appears much closer than its actual position in terms of depth. This is similar to what happens when one fails to account for the refraction model and hence, there is larger error in depth measurement as compared to the lateral error measurement.

Finally, there is some natural variation between people in terms of the value of $\epsilon_{r}$ for the same tissue type. In our evaluation we use the average value of $\epsilon_{r}$. We want to understand how much of an error can the use of this value $\epsilon_{r}$ introduce. We change the value of $\epsilon_{r}$ by up to $10 \%$, which is similar to the natural variation observed in past work [54]. We report the localization error as a function of the change in Fig. 9. As can be seen, the error increases as a function of perturbation of this value. However, even when the change in $\epsilon_{r}$ is $10 \%$, the error in positioning the in-body device is less than $2.5 \mathrm{~cm}$. This shows that the natural variation of human beings can be accommodated by ReMix's design. In addition to that, it also indicates that there is a potential for improving the accuracy by customizing the parameters for each patient. 


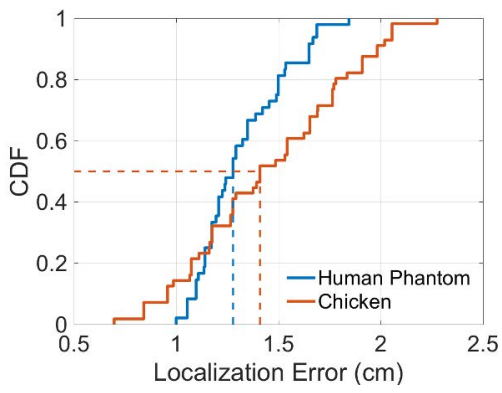

(a) Localization Error

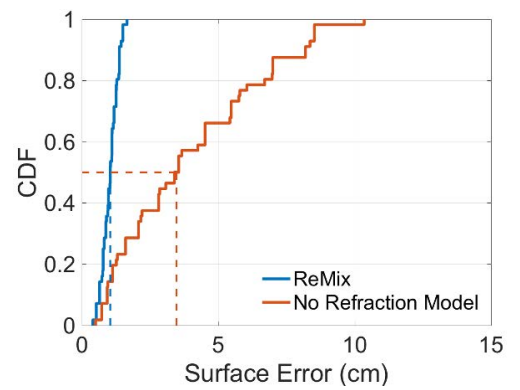

(b) Effect of Refraction Model

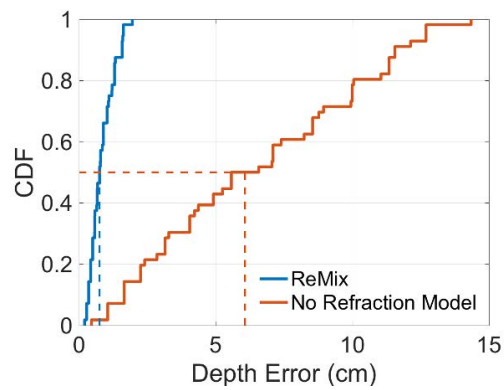

$$
15
$$

Figure 10-ReMix Localization Results: ReMix can achieve high localization accuracy of $1.3 \mathrm{~cm}$ in human phantom and $1.4 \mathrm{~cm}$ in chicken.

\section{Limitations AND FUTURE WORK}

Finally, a few points are worth elaborating:

- ReMix made a few approximations, like grouping skin and muscle in a single layer to reduce model complexity, and using an average value for $\epsilon_{r}$ though different people may have mild variations. In making these assumptions, we strived to balance model complexity with the accuracy requirements. We believe these approximations are acceptable for applications like smart capsules. Future work can extend the model to eliminate these approximations and address applications with more stringent localization requirements (e.g., mm level accuracy for tumor localization in radiation therapy).

- We made our best attempt to emulate human tissues by using phantoms and dead animal tissues for our evaluation, but we did not conduct measurements in live animals. While the analysis and experiments should stand to be correct in living tissue, experiments in live animals fall in the realm of future work.

- In medical applications, typically, there is availability of side channel information, like one-time MRI scans, that can throw more light on the exact composition of the human body. ReMix does not rely on such extra information, but future work that targets specific applications with more stringent requirements might benefit from multi-modal input data.

\section{Conclusion}

We present ReMix, the first system to demonstrate deeptissue backscatter communication and localization. ReMix achieves this goal using two design principles. First, it uses circuit non-linearities to shift the frequency of the backscatter signal to avoid interference from surface reflections. Second, it presents a time-of-flight localization algorithm that accounts for in-body signal refraction. Experiments with animal tissues and human phantoms demonstrate ReMix's effectiveness.

\section{Appendix}

Lemma: If an EM wave travels through $L$ parallel layers, each with thickness, $l_{i}, i=1, \ldots, L$; then the phase accumulated by the EM wave is independent of the order of the layers.
Proof: Consider a 2-D system where a wave propagates in the $X Y$ plane (similar to Fig. 5) from $X_{T}=\left(x_{T}, y_{T}\right)$ to $x_{R}=\left(x_{R}, y_{R}\right)$. Furthermore, without loss of generality, assume that the signal travels through $L$ layers, stacked on top of each other along the $Y$ axis. For this proof, we leverage the concept of wave vectors. Wave vector, $\vec{k}=\left(k_{x}, k_{y}\right)$, is a vector that points in the direction of wave propagation and its magnitude is given by, $k_{x}^{2}+k_{y}^{2}=\left(2 \pi \frac{f}{v}\right)^{2}$, where $v=c / \sqrt{\epsilon_{r} \mu_{r}}$ is the speed of light in the material. Further, if the wave propagation path is denoted as another vector, $\vec{r}=\left(r_{x}, r_{y}\right)$, then the phase accumulated by the wave in travelling through $\vec{r}$ is: $\operatorname{Re}(\vec{k} \cdot \vec{r})$.

For layer $i$, let the wave vector be $\vec{k}_{i}=\left(k_{x i}, k_{y i}\right)$, the propagation path be $\vec{r}_{i}=\left(r_{x i}, r_{y i}\right)$, and the EM parameters be $\left(\epsilon_{r i}, \mu_{r i}\right)$. Then, the phase accumulated by the wave is, $\phi=$ $\sum_{i=1}^{L} \operatorname{Re}\left(\overrightarrow{k_{i}} \cdot \vec{r}_{i}\right)$. Now, observe $r_{y i}=l_{i}$, since the total path along the $Y$-axis in each layer is the depth of the layer. Also, phase continuity at interfaces ensures that, $k_{x i}=k_{x j}=k_{x}$ for all $(i, j)$ [47]. Finally, $\sum_{i=1}^{L} r_{x i}=x_{R}-x_{T}$, since we are considering the phase for propagation from transmitter to receiver. Combining, we have:

$$
\begin{aligned}
\phi & =\sum_{i=1}^{L} \operatorname{Re}\left(k_{x}\right) r_{x i}+\operatorname{Re}\left(k_{y} i\right) l_{i} \\
& =\operatorname{Re}\left(k_{x}\right) \sum_{i=1}^{L} r_{x i}+\sum_{i=1}^{L}\left(2 \pi \frac{f}{c}\right)^{2} \epsilon_{r i} \mu_{r i} l_{i} \\
& =\operatorname{Re}\left(k_{x}\right)\left(x_{R}-x_{T}\right)+\sum_{i=1}^{L}\left(2 \pi \frac{f}{c}\right)^{2} \epsilon_{r i} \mu_{r i} l_{i}
\end{aligned}
$$

Notice that Eq. 20 just depends on the depth of each layer and is independent of the order. This implies that the phase accumulated by an EM wave in traversing parallel stacked layers is independent of the order of stacked layers.

Acknowledgements- We thank the members of the NETMIT group, anonymous reviewers and our shepherd, Kate Lin, for their insightful comments and feedback. We thank Hariharan Rahul for his help with writing the paper. We are grateful to Sumitomo Heavy Industries, Ltd for funding this research and the Microsoft Research PhD fellowship for supporting Deepak Vasisht. 


\section{REFERENCES}

[1] A. M. A. A. T. Mobashsher. Artificial human phantoms: Human proxy in testing microwave apparatus that have electromagnetic interaction with the human body. ArXiv, 2015.

[2] A. Abid, Jonathan M. O'Brien, T. Bensel, C. Cleveland, L. Booth, B. R. Smith, R. Langer, and G. Traverso. Wireless power transfer to millimetersized gastrointestinal electronics validated in a swine model. Nature Scientific Reports, 2017.

[3] American Society for Gastrointestinal Endoscopy. Wireless capsule endoscopy, 2013. https://www.asge.org/docs/default-source/importfiles/ assets/0/73730/c4d44578-c3d0-4583-9949-b15f3e8537e0.pdf?sfvrsn= 4.

[4] S. M. Aziz, M. Grcic, and T. Vaithianathan. A Real-Time Tracking System for an Endoscopic Capsule using Multiple Magnetic Sensors. Springer Berlin Heidelberg, 2008.

[5] M. R. Basar, F. Malek, K. M. Juni, M. S. Idris, and M. I. M. Saleh. Ingestible wireless capsule technology: A review of development and future indication. International Journal of Antennas and Propagation, 2012.

[6] D. Bharadia, K. R. Joshi, M. Kotaru, and S. Katti. BackFi: High Throughput WiFi Backscatter. ACM SIGCOMM, 2015.

[7] J. Brooks. Swedish workers implanted with microchips to replace cash cards and id passes. Independent UK, 2017.

[8] R. Chandra, A. J. Johansson, and F. Tufvesson. Localization of an rf source inside the human body for wireless capsule endoscopy. BodyNets, 2013.

[9] X. Chen, X. Zhang, L. Zhang, X. Li, N. Qi, H. Jiang, and Z. Wang. A wireless capsule endoscope system with low-power controlling and processing asic. IEEE Transactions on Biomedical Circuits and Systems, 2009.

[10] B. G. Colpitts and G. Boiteau. Harmonic radar transceiver design: miniature tags for insect tracking. IEEE Transactions on Antennas and Propagation, 2004.

[11] W. contributors. Eb/n0 - wikipedia, the free encyclopedia, 2017. https: //en.wikipedia.org/w/index.php?title=Eb/N0\&oldid=809750730.

[12] W. contributors. Magnetic dipole - wikipedia, the free encyclopedia, 2017. https://en.wikipedia.org/w/index.php?title=Magnetic_dipole\& oldid $=811519977$.

[13] J. R. Cook, R. R. Bouchard, and S. Y. Emelianov. Tissue-mimicking phantoms for photoacoustic and ultrasonic imaging. Biomedical Optics Express, 2011.

[14] A. B. de GonzÃąlez and S. Darby. Risk of cancer from diagnostic x-rays: estimates for the uk and 14 other countries. The Lancet, 2004

[15] I. Dietlicher, M. Casiraghi, C. Ares, A. Bolsi, D. Weber, A. Lomax, and F. Albertini. Experimental measurement with an anthropomorphic phantom of the proton dose distribution in the presence of metal implants. PTCOG, 2014.

[16] I. Dove. Analysis of radio propagation inside the human body for in-body localization purposes. Master's thesis, University of Twente, 2014.

[17] Ettus Research. USRP X310. https://www.ettus.com/product/details/ X310-KIT.

[18] FCC. FCC Publication 703867, 2017. https://apps.fcc.gov/oetcf/kdb/ forms/FTSSearchResultPage.cfm?id=27023\&switch $=P$.

[19] K. R. Foster and J. Jaeger. Rfid inside. IEEE Spectrum, 2007.

[20] H. Gomes and N. B. Carvalho. Rfid for location proposes based on the intermodulation distortion. Sensors \& Transducers, 2009.

[21] H. C. Gomes and N. B. Carvalho. The use of intermodulation distortion for the design of passive rfid. In 2007 European Radar Conference, 2007.

[22] J. Hou, Y. Zhu, L. Zhang, Y. Fu, F. Zhao, L. Yang, and G. Rong. Design and implementation of a high resolution localization system for in-vivo capsule endoscopy. In 2009 Eighth IEEE International Conference on Dependable, Autonomic and Secure Computing, 2009.
[23] C. Hu, M. Q. Meng, and M. Mandal. Efficient magnetic localization and orientation technique for capsule endoscopy. In 2005 IEEE/RSJ International Conference on Intelligent Robots and Systems, 2005.

[24] P. Hu, P. Zhang, M. Rostami, and D. Ganesan. Braidio: An Integrated Active-Passive Radio for Mobile Devices with Asymmetric Energy Budgets. ACM SIGCOMM, 2016.

[25] H. J. Huisman, J. J. Fütterer, E. N. J. T. van Lin, A. Welmers, T. W. J. Scheenen, J. A. van Dalen, A. G. Visser, J. A. Witjes, and J. O. Barentsz. Prostate cancer: Precision of integrating functional $\mathrm{mr}$ imaging with radiation therapy treatment by using fiducial gold markers. Radiology, 2005.

[26] Institute of Applied Physics. Dielectric Properties of Body Tissues. http://niremf.ifac.cnr.it/tissprop/htmlclie/htmlclie.php.

[27] T. Instruments. ISM-Band and Short Range Device Regulatory Compliance Overview, 2005. http://www.ti.com/lit/an/swra048/swra048.pdf.

[28] K. Ito, K. Furuya, Y. Okano, and L. Hamada. Development and characteristics of a biological tissue-equivalent phantom for microwaves. Electronics and Communications in Japan (Part I: Communications), 2001.

[29] E. Kanal, A. J. Barkovich, C. Bell, J. P. Borgstede, W. G. B. Jr, J. W. Froelich, J. R. Gimbel, J. W. Gosbee, E. Kuhni-Kaminski, P. A. Larson, J. W. L. Jr, J. Nyenhuis, D. J. Schaefer, E. A. Sebek, J. Weinreb, B. L. Wilkoff, T. O. Woods, L. Lucey, and D. Hernandez. Acr guidance document on mr safe practices: 2013. Journal Of Magnetic Resonance Imaging, 2013.

[30] B. Kellogg, V. Talla, S. Gollakota, and J. R. Smith. Passive wi-fi: Bringing low power to wi-fi transmissions. USENIX NSDI, 2016.

[31] J. Kim and Y. Rahmat-Samii. Implanted antennas inside a human body: simulations, designs, and characterizations. IEEE Transactions on $\mathrm{Mi}$ crowave Theory and Techniques, 2004.

[32] R. W. P. King, G. S. Smith, M. Owens, and T. T. Wu. Antennas in matter: Fundamentals, theory, and applications. NASA STI/Recon Technical Report A, 81, 1981.

[33] M. Kotaru, K. Joshi, D. Bharadia, and S. Katti. Spotfi: Decimeter level localization using wifi. ACM SIGCOMM, 2015.

[34] H. D. Kubo and B. C. Hill. Respiration gated radiotherapy treatment: a technical study. Physics in Medicine and Biology, 1996.

[35] D. Kurup, GuIILnter Vermeeren, Emmeric Tanghe, W. Joseph, and L. Martens. In-to-out body antenna-independent path loss model for multilayered tissues and heterogeneous medium. IEEE Sensors, 2014.

[36] M. Lazebnik, E. L. Madsen, G. R. Frank, and S. C. Hagness. Tissuemimicking phantom materials for narrowband and ultrawideband microwave applications. Physics in Medicine and Biology, 2005.

[37] V. Liu, A. Parks, V. Talla, S. Gollakota, D. Wetherall, and J. R. Smith. Ambient Backscatter: Wireless Communication out of Thin Air. ACM SIGCOMM, 2013.

[38] R. Lodato, V. Lopresto, R. Pinto, and G. Marrocco. Numerical and experimental characterization of through-the-body uhf-rfid links for passive tags implanted into human limbs. IEEE Transactions on Antennas and Propagation, 2014.

[39] A. Ma and A. S. Y. Poon. Midfield wireless power transfer for bioelectronics. IEEE Circuits and Systems Magazine, 2015.

[40] D. Manteuffel and M. Grimm. Localization of a functional capsule for wireless neuro-endoscopy. In 2012 IEEE Topical Conference on Biomedical Wireless Technologies, Networks, and Sensing Systems (BioWireleSS), 2012.

[41] A. Masters and K. Michael. Lend me your arms: The use and implications of humancentric rfid. Electronic Commerce Research and Applications, 2007.

[42] H. J. Meyer, N. Chansue, and F. Monticelli. Implantation of radio frequency identification device (rfid) microchip in disaster victim identification (dvi). Forensic Science International, 2006. 
[43] K. Michael. Rfid/nfc implants for bitcoin transactions. IEEE Consumer Electronics Magazine, 2016.

[44] B. J. Mohammed, A. M. Abbosh, S. Mustafa, and D. Ireland. Microwave system for head imaging. IEEE Transactions on Instrumentation and Measurement, 2014.

[45] C. Oancea, K. Shipulin, G. Mytsin, A. Molokanov, D. Niculae, I. Ambrozová, and M. Davídková. Effect of titanium dental implants on proton therapy delivered for head tumors: experimental validation using an anthropomorphic head phantom. Journal of Instrumentation, 2017

[46] T. Onishi and S. Uebayashi. Biological Tissue-equivalent Phantoms Usable in Broadband Frequency Range. NTT DoCoMo Technical Journal, 2006.

[47] S. J. Orfanidis. Electromagnetic waves and antennas. Rutgers University New Brunswick, NJ, 2002.

[48] G. Ou, N. Shahidi, C. Galorport, O. Takach, T. Lee, and R. Enns. Effect of longer battery life on small bowel capsule endoscopy. World Journal of Gastroenterology, 2015.

[49] D. M. Pham and S. M. Aziz. A real-time localization system for an endoscopic capsule using magnetic sensors. IEEE Sensors, 2014.

[50] K. Rasilainen, J. Ilvonen, A. Lehtovuori, J. M. Hannula, and V. Viikari. On design and evaluation of harmonic transponders. IEEE Transactions on Antennas and Propagation, 2015.

[51] S. Y. Semenov, A. E. Bulyshev, A. Abubakar, V. G. Posukh, Y. E. Sizov, A. E. Souvorov, P. M. van den Berg, and T. C. Williams. Microwavetomographic imaging of the high dielectric-contrast objects using different image-reconstruction approaches. IEEE Transactions on Microwave Theory and Techniques, 2005.

[52] Skyworks. SMS7630 Series. http://www.skyworksinc.com/Product/511/ SMS7630_Series?IsProduct=true.

[53] P. R. Stauffer, F. Rossetto, M. Prakash, D. G. Neuman, and T. Lee. Phantom and animal tissues for modelling the electrical properties of human liver. International Journal of Hyperthermia, 2003.

[54] A. Surowiec, S. S. Stuchly, L. Eidus, and A. Swarup. In vitro dielectric properties of human tissues at radiofrequencies. Physics in Medicine and Biology, 1987.

[55] Q. Tang, S. K. S. Gupta, and L. Schwiebert. Ber performance analysis of an on-off keying based minimum energy coding for energy constrained wireless sensor applications. In IEEE International Conference on Communications, 2005.

[56] Taoglas. PC 30 Antenna. http://www.taoglas.com/product/ pc30-2g3g-cellular-fr4-pcb-antenna-mmcxmra- $2 /$.

[57] D. Tse and P. Vishwanath. Fundamentals of Wireless Communications. Cambridge University Press, 2005.

[58] I. Umay, B. Fidan, and B. Barshan. Localization and tracking of implantable biomedical sensors. IEEE Sensors, 2017.

[59] I. Umay, B. Fidan, and M. R. YÃijce. Endoscopic capsule localization with unknown signal propagation coefficients. In 2015 International Conference on Advanced Robotics (ICAR), 2015.

[60] D. Vasisht, S. Kumar, and D. Katabi. Decimeter-Level Localization with a Single WiFi Access Point. USENIX NSDI, 2016.

[61] J. Wang, D. Vasisht, and D. Katabi. Rf-idraw: Virtual touch screen in the air using rf signals. ACM SIGCOMM, 2014.

[62] Y. Wang, R. Fu, Y. Ye, U. Khan, and K. Pahlavan. Performance bounds for rf positioning of endoscopy camera capsules. In 2011 IEEE Topical Conference on Biomedical Wireless Technologies, Networks, and Sensing Systems, 2011.

[63] J. Xiong and K. Jamieson. ArrayTrack: A Fine-Grained Indoor Location System. USENIX NSDI, 2013.

[64] Y. Ye and K. Pahlavan. Accuracy bounds for and rss and toa based rf localization in capsule endoscopy. 2011.

[65] M. R. Yuce and T. Dissanayake. Easy-to-swallow wireless telemetry. IEEE Microwave Magazine, 2012.
[66] L. Zhang, Y. Zhu, T. Mo, J. Hou, and H. Hu. Design of 3d positioning algorithm based on rfid receiver array for in vivo micro-robot. In IEEE International Conference on Dependable, Autonomic and Secure Computing, 2009.

[67] L. Zhang, Y. Zhu, T. Mo, J. Hou, and G. Rong. Design and implementation of $3 \mathrm{~d}$ positioning algorithms based on rf signal radiation patterns for in vivo micro-robot. International Conference on Body Sensor Networks, 2010.

[68] P. Zhang, D. Bharadia, K. Joshi, and S. Katti. HitchHike: Practical Backscatter Using Commodity WiFi. ACM SenSys, 2016. 